\title{
Non-Dystrophic Myotonia
}

National Cancer Institute

\section{Source}

National Cancer Institute. Non-Dystrophic Myotonia. NCI Thesaurus. Code C122787.

A group of rare skeletal muscle ion-channel disorders caused by genetic mutations in the sodium and chloride channel genes. It is characterized by altered membrane excitability resulting in skeletal muscle stiffness. This group of myotonias is distinct from myotonic dystrophy because of the absence of systemic features or progressive weakness. 